\title{
News from BESIII
}

\author{
Stephen Lars Olsen ${ }^{* \dagger}$ \\ Center for Underground Physics, Institute for Basic Science, Daejeon 34047, KOREA \\ E-mail: solsensnu@gmail.com
}

\begin{abstract}
I present a summary of recent results from the BESIII experiment that relate to the study of the enigmatic $Y(4260)$ charmonium-like resonance. First I review the considerable body of existing experimental material on the $Y(4260)$ and briefly describe some of the proposed theoretical models for its underlying structure. I then describe critical measurements that can be done at BESIII with a data sample of double-tagged $e^{+} e^{-} \rightarrow \pi^{ \pm} D^{0} \bar{D}^{0}\left(\pi^{\mp}\right)$ events, where the $\pi^{\mp}$ is not detected but its four-momentum is inferred from energy-momentum conservation. The well established D-wave dominance of the $D_{1}(2420) \rightarrow D^{*} \pi$ decay vertex provides a powerful diagnostic tool that can be used to discriminate between the $D \bar{D}_{1}(2420)$ molecular picture and other proposed models. However, this discrimination can only be accomplished in the context of a complete three-dimensional amplitude analysis.
\end{abstract}

55th International Winter Meeting on Nuclear Physics

23-27 January, 2017

Bormio, Italy

\footnotetext{
* Speaker.

${ }^{\dagger}$ Work supported by the Institute for Basic Science (Korea) under project code: IBS-R016-D1.
} 


\section{Introduction}

The BESIII detector is a nearly $4 \pi$ solid-angle, multipurpose spectrometer that is based on a large, 1 Tesla superconducting solenoid magnet and operates at the BEPCII double-ring $e^{+} e^{-}$ collider in Beijing China. The c.m, energy range accessed by BEPCII, $2.0 \leq E_{\mathrm{cm}} \leq 4.6 \mathrm{GeV}$, covers thresholds that range from $\Lambda \bar{\Lambda}$ to $\Lambda_{c}^{+} \Lambda_{c}^{-}$production and include those for $\tau^{+} \tau^{-}$and pairs of open-charmed mesons. As a result, BESIII has an enormously rich scientific program, with a large number of unique opportunities for doing forefront measurements in a wide variety of subjects. It would be impossible to do justice to all of the new results being produced by the BESIII team in a short report like this. Instead I focus on results and short-term prospects in one area that is of particular interest to me: the increasingly mysterious $Y(4260)$ charmonium-like meson.

The $Y(4260)$ has attracted considerable attention; the BaBar [1] paper reporting its discovery ranks among that experiment's most highly cited papers. ${ }^{1}$ The $Y(4260)$ resonance: was discovered more than a decade ago; has $J^{P C}$ quantum numbers that are firmly established as $1^{--}$; has many interesting decay modes that have been identified and studied in detail; and has been the subject of many hundreds of theoretical papers. Nevertheless, its underlying nature still remains an intriguing puzzle. In fact, it seems that the more we learn about it, the more the mysterious it is.

Fortunately the BESIII experiment is uniquely well suited for detailed experimental studies of the $Y(4260)$ that may provide the key to solving its associated puzzles and, perhaps, provide some profound insights into nature of the other $X Y Z$ mesons and better characterize the longdistance properties of Quantum Chromodynamics (QCD). In this report I summarize what is currently known about the experimental properties of the $Y(4260)$, briefly list the various theoretical explanations for this state, and then describe possible measurements that could be done with BESIII that could confront these theories. We start with a summary of the experimental status of the $Y(4260)$.

\section{What is known about the $Y(4260)$}

The $Y(4260)$ was discovered by the BaBar experimental group as a striking and unexpected peak in the $\pi^{+} \pi^{-} J / \psi$ invariant mass distribution produced in $e^{+} e^{-}$collisions at $E_{\mathrm{cm}}=10.58 \mathrm{GeV}$ via the initial state radiation (isr) process $e^{+} e^{-} \rightarrow \gamma_{\text {isr }} \pi^{+} \pi^{-} J / \psi$ [1]. BaBar's most recent measurements of the Born cross sections for $e^{+} e^{-} \rightarrow \pi^{+} \pi^{-} J / \psi$ [2] in the vicinity of the $Y(4260)$ peak are shown in Fig. 1a. This peak was quickly confirmed by the CLEO [3] and Belle groups [4]. The most recent Belle measurements [5] of $\sigma\left(e^{+} e^{-} \rightarrow \pi^{+} \pi^{-} J / \psi\right)$ in the $Y(4260)$ region, based on their full data set, are shown in Fig. 1b, where the close similarity to the BaBar signal is apparent. The PDG-2016 [6] world-average of the mass and width values, determined from Babar, CLEO and Belle from fits of a single Breit wigner (BW) resonance line shape to the $Y(4260)$ peaks in their data are:

$$
\begin{aligned}
M(Y(4260)) & =4251 \pm 9 \mathrm{MeV} \\
\Gamma(Y(4260)) & =120 \pm 12 \mathrm{MeV} .
\end{aligned}
$$

\footnotetext{
${ }^{1}$ Among the BaBar group members responsible for the $Y(4260)$ discovery, were long-time BES collaborator Xinchou Lou and BES alumnus Shuwei Ye.
} 

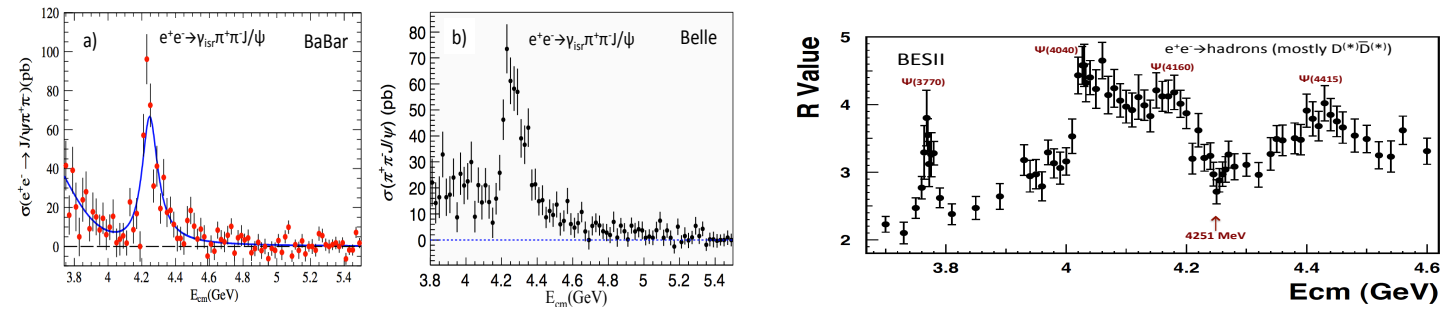

Figure 1: a) The data points show the Born cross sections Figure 2: $R=\sigma_{\mathrm{tot}}\left(e^{+} e^{-} \rightarrow\right.$ hadrons $) / \sigma_{\mathrm{QED}}\left(e^{+} e^{-} \rightarrow\right.$ for $e^{+} e^{-} \rightarrow \pi^{+} \pi^{-} J / \psi$, measured via the initial-state radia- $\left.\mu^{+} \mu^{-}\right)$, where $\sigma_{\mathrm{QED}}\left(e^{+} e^{-} \rightarrow \mu^{+} \mu^{-}\right)=86.85 \mathrm{nb} / s(s$ tion process $e^{+} e^{-} \rightarrow \gamma_{\text {isr }} \pi^{+} \pi^{-} J / \psi$ by BaBar [2]. The curve in $\mathrm{GeV}^{2}$ ), from BESII [7]. The structures above $R \simeq 2$ show results of a fit that used a single BW resonance to rep- are due to the indicated $1^{--}$charmonium mesons deresent the $Y(4260)$ resonance plus a linear background term. caying to $D \bar{D}, D \bar{D}^{*}$ or $D^{*} \bar{D}^{*}$ open-charm final states. b) Belle measurements of the same cross sections [5]. The position of the $Y(4260)$ peak, indicated by an arrow, is located at a local minimum in the cross section.

The production mode of the $Y(4260)$ ensures that its $J^{P C}$ quantum numbers are the same as those of the photon, i.e. $1^{--}$. Its discovery decay mode, $Y(4260) \rightarrow \pi^{+} \pi^{-} J / \psi$, provides strong evidence that its constituents must contain a $c \bar{c}$ quark pair. However, all of the $1^{--} c \bar{c}$ charmonium levels with mass below $4500 \mathrm{MeV}$ have already been assigned to well established $1^{--}$resonances that are seen in the total cross section for $e^{+} e^{-} \rightarrow$ hadrons between 2.6 and $4.6 \mathrm{GeV}[8,7]$. In addition, even though its mass is well above all of the $D^{(*)} \bar{D}^{(*)}$ open-charmed-meson mass thresholds, there is no evidence for its decay to pairs of open-charmed mesons in the total cross section for $e^{+} e^{-}$annihilations into hadrons. BESII measurements of $\sigma_{\mathrm{tot}}\left(e^{+} e^{-} \rightarrow\right.$ hadrons $)$ at c.m. energies between 3.7 and $4.6 \mathrm{GeV}$, shown in Fig. 2, exhibit considerable structure that is well described by the production and decay to open-charmed mesons of the established $\psi(3770)$, $\psi(4040), \psi(4160)$ and $\psi(4415) 1^{--}$charmonium states [9]. The strong signals for these states in $\sigma_{\mathrm{tot}}\left(e^{+} e^{-} \rightarrow\right.$ hadrons $)$ in Fig. 1a, plus their absence in the $M\left(\pi^{+} \pi^{-} J / \psi\right)$ invariant mass distribution in Fig. 1b, reflect the expected strong dominance of fall-apart decays to open-charmed-meson pairs over OZI-rule-suppressed decays to hidden-charm final states that is characteristic of aboveopen-charm-threshold charmonium states. In contrast, the absence of any sign of $Y(4260)$ decays to charmed mesons in $\sigma_{\mathrm{tot}}\left(e^{+} e^{-} \rightarrow\right.$ hadrons $)$ plus its strong signal in the $\pi^{+} \pi^{-} J / \psi$ decay channel is opposite to expectations for charmonium. As a result, there has been considerable theoretical speculation that the $Y(4260)$ might be some kind of a multi-quark meson or a $c \bar{c}$-gluon hybrid state [10].

\subsection{BESIII as a "Y (4260)-Factory"}

The BaBar and Belle results on the $Y(4260)$ relied on production of this state via the isr process. This process has the advantage of sampling many $e^{+} e^{-}$c.m. energies at once, but is limited by a severe, order $\alpha_{\mathrm{QED}}$, luminosity penalty associated with the radiation of a hard photon. For detailed studies of this state, the BESIII experiment has the advantage of operating at and near c.m. energies corresponding to the resonance peak, where it can produce $Y(4260)$ 's directly, i.e. functioning as a " $Y(4260)$-factory." In this mode of operation, large event samples can be accumulated near the peak of the resonance. On the other hand, lineshape measurements of the resonance parameters and the separation of resonance signals from underlying non-resonant backgrounds re- 
quire time-consuming energy-by-energy scans.

\subsubsection{BESIII discoveries of the $Z_{c}(3900)$ and the $Z_{c}(4020)$}

BESIII's first data-taking run in this energy range accumulated a $525 \mathrm{pb}^{-1}$ data sample at $4260 \mathrm{MeV}$ in which they found $1477 \pi^{+} \pi^{-} J / \psi$ events that included 307 events of the type $e^{+} e^{-} \rightarrow$ $\pi^{\mp} Z_{c}(3900)^{ \pm} ; Z_{c}(3900)^{ \pm} \rightarrow \pi^{ \pm} J / \psi[11]$, where the $Z_{c}(3900)^{ \pm}$is a relatively narrow, resonancelike structure with non-zero electric charge. Shortly thereafter, a study of $e^{+} e^{-} \rightarrow \pi^{ \pm}\left(D \bar{D}^{*}\right)^{\mp}$ found a strong $\left(D \bar{D}^{*}\right)^{\mp}$ peak at a similar mass, called the $Z_{c}$ (3885) [12], a study of $e^{+} e^{-} \rightarrow \pi^{+} \pi^{-} h_{c}$ found a $\pi^{\mp} h_{c}$ peak near $4020 \mathrm{MeV}$ called the $Z_{c}$ (4020) [13], and a study of $e^{+} e^{-} \rightarrow \pi^{ \pm}\left(D^{*} \bar{D}^{*}\right)^{\mp}$ found a $\left(D^{*} \bar{D}^{*}\right)^{\mp}$ peak near $4025 \mathrm{MeV}$, called the $Z_{c}(4025)$ [14]. We briefly describe these discoveries in the following.

The $Z_{c}(3900)$ : Figure 3 shows the distribution of the larger of the $\pi^{+} J / \psi$ and $\pi^{-} J / \psi /$ invariant mass combinations in $Y(4260) \rightarrow \pi^{+} \pi^{-} J / \psi$ events in a $525 \mathrm{pb}^{-1}$ BESIII data sample accumulated at $E_{\mathrm{cm}}=4.260 \mathrm{GeV}$ [11]. Here a distinct peak, called the $Z_{c}(3900)$, is evident near $3900 \mathrm{MeV}$. A fit using a BW amplitude to represent the $\pi^{ \pm} J / \psi$ mass peak and an incoherent phase-space-like function to represent the non-resonant background gave mass and width values of $M\left(Z_{c}(3900)\right)=3899.0 \pm 6.1 \mathrm{MeV}$ and $\Gamma\left(Z_{c}(3900)\right)=46 \pm 22 \mathrm{MeV}$, which is $\sim 24 \mathrm{MeV}$ above the $m_{D^{*+}}+m_{\bar{D}^{0}}\left(\right.$ or $\left.m_{D^{+}}+m_{\bar{D}^{* 0}}\right)$ threshold. The $Z_{c}(3900)$ was observed by Belle in isr data at about the same time [5].
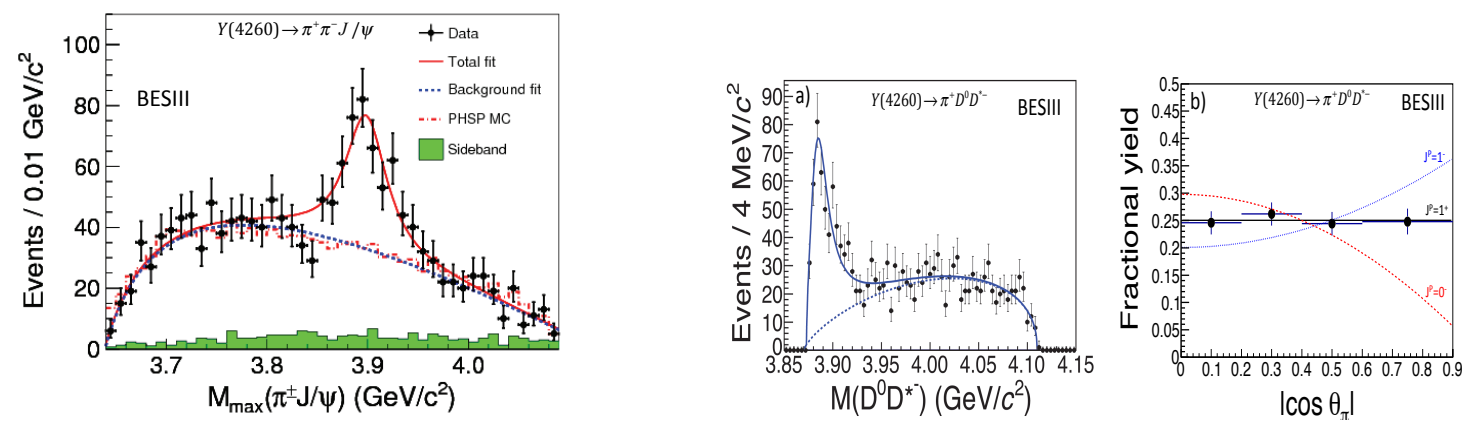

Figure 3: Distribution of the larger of the two $\pi^{ \pm} J / \psi$ Figure 4: a) The $D^{0} D^{*-}$ invariant mass distribution in masses in $e^{+} e^{-} \rightarrow \pi^{+} \pi^{-} J / \psi$ events collected in the $e^{+} e^{-} \rightarrow \pi^{+} D^{0} D^{*-}$ events at $E_{\mathrm{cm}}=4.260 \mathrm{GeV}$ [12]. The solid BESIII detector at $\sqrt{s}=4.260 \mathrm{GeV}$. The histogram curve shows a fit to the data with a threshold-modified BW amshows the level of the non- $J / \psi$ background, which is plitude plus an incoherent phase-space-like background (dashed determined from $J / \psi$ mass sideband events. The figure curve). b) The $Z_{c}(3885)$ production angle distribution with exis from ref. [11]. pectations for different $J^{P}$ assignments [12].

A BESIII study of the $D^{0} D^{*-}$ systems produced in $e^{+} e^{-} \rightarrow \pi^{+} D^{0} D^{*-}$ final states in the same data sample [12] found the very strong near-threshold peak in the $D^{0} D^{*-}$ and invariant mass distribution shown in Fig. 4a. The solid curve in the figure shows the results of a fit to the data with a threshold-modified BW amplitude to represent the peak and an incoherent phase-space-like function to represent the background. The same analysis found a similar peak in the $D^{-} D^{* 0}$ invariant mass distribution in $e^{+} e^{-} \rightarrow \pi^{+} D^{-} D^{* 0}$ events. The masses and widths from the two channels are consistent and their average values are $M=3883.9 \pm 4.5 \mathrm{MeV}$ and $\Gamma=24.8 \pm 12 \mathrm{MeV}$,

Since the mass is $\simeq 2 \sigma$ lower than the $Z_{c}(3900)$ mass reported in ref. [11], BESIII cautiously named this $D \bar{D}^{*}$ state the $Z_{c}(3885)$. In the mass determinations of both the $Z_{c}(3885)$ and $Z_{c}(3900)$, 
effects of possible interference with a coherent component of the non-resonant background are ignored, an approximation that can bias mass measurements by amounts comparable to the resonance widths; this effect could account for the different mass values and it seems highly likely that the $Z_{c}(3885)$ is the $Z_{c}$ (3900) in a different decay channel. If this is the case, the partial width for $Z_{c}(3900) \rightarrow D \bar{D}^{*}$ decays is $6.2 \pm 2.9$ times larger than that for $Z_{c}(3900) \rightarrow \pi^{+} J / \psi$, which is small compared to open-charm $v s$. hidden-charm decay-width ratios for established charmonium states above the open-charm threshold, such as the $\psi(3770)$ and $\psi(4040)$, where corresponding ratios are measured to be more than an order-of-magnitude larger [6].

The strong $Y(4260) \rightarrow \pi^{+} Z_{c}(3900) ; Z(3885) \rightarrow D \bar{D}^{*}$ signal enabled the BESIII group to determine its $J^{P}$ quantum numbers from the dependence of its production on $\theta_{\pi}$, the "bachelor" $\pi^{+}$ production angle relative to the beam direction in the $e^{+} e^{-} \mathrm{cm}$ system. For $J^{P}=0^{-}, d N / d\left|\cos \theta_{\pi}\right|$ should go as $\sin ^{2} \theta_{\pi}$; for $1^{-}$it should follow $1+\cos ^{2} \theta_{\pi}$ and for $1^{+}$it should be flat $\left(0^{+}\right.$is forbidden by Parity). Figure $4 \mathrm{~b}$ shows the efficiency-corrected $Z_{c}(3885)$ signal yield as a function of $\left|\cos \theta_{\pi}\right|$, together with expectations for $J^{P}=0^{+}$(dashed red), $1^{-}$(dotted blue) and $J^{P}=1^{+}$. The $J^{P}=1^{+}$ assignment is clearly preferred and the $0^{-}$and $1^{-}$assignments are ruled out with high confidence. ${ }^{2}$

BESIII also reported neutral counterparts of the $Z_{c}(3900)$ in the $\pi^{0} J / \psi$ channel in $e^{+} e^{-} \rightarrow$ $\pi^{0} \pi^{0} J / \psi$ events [16], and the $D^{+} D^{*-}$ and $D^{0} \bar{D}^{* 0}$ channels in $e^{+} e^{-} \rightarrow \pi^{0}\left(D \bar{D}^{*}\right)^{0}$ events [17], with mass and width values that are in good agreement with the charged $Z_{c}(3900)$ state measurements. The relative signal yields in the charged and neutral channels are consistent with expectations based on isospin conservation.

The $Z_{c}(4020)$ : With data accumulated at c.m. energies at 4260 and $4360 \mathrm{MeV}$ and nearby energies, BESIII made a study of $\pi^{+} \pi^{-} h_{c}(1 \mathrm{P})$ final states [13]. Exclusive $h_{c}(1 \mathrm{P})$ decays were detected via the $h_{c} \rightarrow \gamma \eta_{c}$ transition, where the $\eta_{c}$ mesons were reconstructed in one of 16 different exclusive hadronic decay channels. With these data, BESIII observed the distinct peak near $4020 \mathrm{MeV}$ in the $M_{\max }\left(\pi^{ \pm} h_{c}\right)$ distribution that is shown in Fig. 5a. A fit to this peak, which the BESIII group called the $Z_{c}(4020)^{+}$, with a signal BW amplitude (assuming $J^{P}=1^{+}$) plus a smooth background, returns a $\sim 9 \sigma$ significance signal with a mass of $M\left(Z_{c}(4020)\right)=4022.9 \pm 2.8 \mathrm{MeV}$, about $5 \mathrm{MeV}$ above $m_{D^{*+}}+m_{\bar{D}^{* 0}}$, and a width of $\Gamma\left(Z_{c}(4020)\right)=7.9 \pm 3.7 \mathrm{MeV}$.

The inset in Fig. 5a shows the result of including a $Z_{c}(3900)^{+} \rightarrow \pi^{+} h_{c}$ term in the fit. In this case, a marginal $\sim 2 \sigma$ signal for $Z_{c}(3900)^{+} \rightarrow \pi^{+} h_{c}$ is seen to the left of the $Z_{c}(4020)$ peak. This translates into an upper limit on the branching fraction for $Z_{c}(3900)^{+} \rightarrow \pi^{+} h_{c}$ decay that is less than that for $Z_{C}(3900)^{+} \rightarrow \pi^{+} J / \psi$ by a factor of five.

BESIII also observed the neutral isospin partner of the $Z_{c}(4020)$ [18]. The $M_{\max }\left(\pi^{0} h_{c}\right)$ distribution for $e^{+} e^{-} \rightarrow \pi^{0} \pi^{0} h_{c}$ events in the same data set that was used used for the $Z_{c}(4020)^{ \pm}$study, shown in Fig. 5b, looks qualitatively like the $M_{\max }\left(\pi^{+} h_{c}\right)$ distribution with a distinct peak near $4020 \mathrm{MeV}$. A fit to the data that includes a BW term with a width fixed at the value measured for the $Z_{c}(4020)^{+}$and floating mass returns a mass of $4023.9 \pm 4.4 \mathrm{MeV}$; this and the signal yield are in good agreement with expectations based on isospin symmetry.

A study of $e^{+} e^{-} \rightarrow \pi^{-} D^{*+} \bar{D}^{* 0}$ events in the $\sqrt{s}=4.260 \mathrm{GeV}$ data sample using a partial reconstruction technique that only required the detection of the bachelor $\pi^{-}$, the $D^{+}$from the

\footnotetext{
${ }^{2} \mathrm{~A}$ recent BESIII partial-wave-analysis of $Y(4260) \rightarrow \pi^{+} \pi^{-} J / \psi$ decays established that the $J^{P}$ of the $Z_{c}(3900) \rightarrow$ $\pi J / \psi$ resonance is $1^{+}$with high confidence [15], thereby making the case for the identification of the $Z_{c}(3885)$ and the $Z_{c}(3900)$ as different decay modes of the same state considerably stronger.
} 

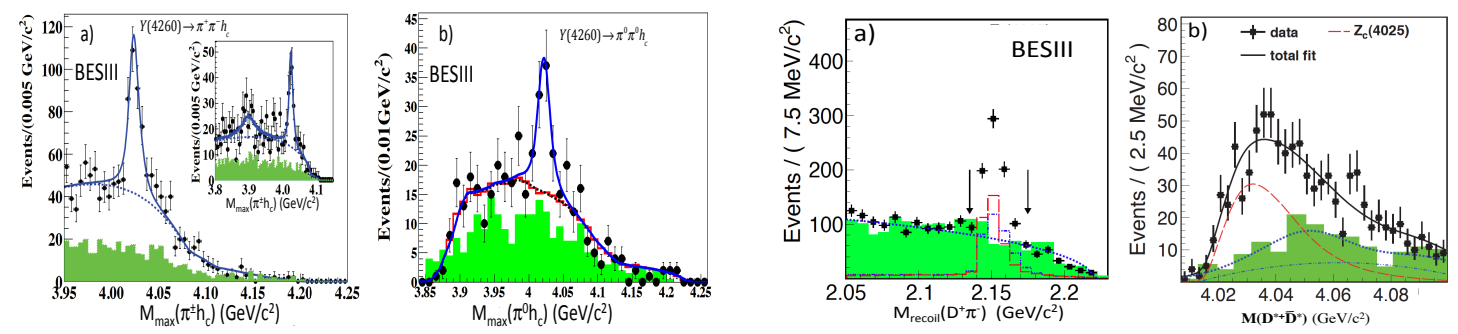

Figure 5: a) The distribution of the larger of the two $\pi^{ \pm} h_{c}$ Figure 6: a) Distribution of masses recoiling against the masses in $e^{+} e^{-} \rightarrow \pi^{+} \pi^{-} h_{c}$ events collected in the BESIII reconstructed $\pi^{-}$and $D^{+}$in $e^{+} e^{-} \rightarrow \pi^{-}\left(D^{*} \bar{D}^{*}\right)^{+}$events detector at $E_{\mathrm{cm}}=4.260$ and $4.360 \mathrm{GeV}$. The inset shows a collected in the BESIII detector at $E_{\mathrm{cm}}=4.260 \mathrm{GeV}$. The larger $M\left(\pi h_{c}\right)$ that includes the $Z_{c}(3900)$ mass region. b) peak near $2.15 \mathrm{GeV}$ corresponds to $e^{+} e^{-} \rightarrow \pi^{-}\left(D^{*+} \bar{D}^{* 0}\right)^{+}$ The $M_{\max }\left(\pi^{0} h_{c}\right)$ distribution for $e^{+} e^{-} \rightarrow \pi^{0} \pi^{0} h_{c}$ events. signal events. b) The $M\left(D^{*+} \bar{D}^{* 0}\right)$ distribution inferred from The histograms show the level of the non- $h_{c}$ background de- the $\pi^{-}$momentum for signal events. The histograms show termined from $h_{c}$ mass sideband events. The curves are de- the level of the combinatoric background determined from scribed in the text. The figures are from refs. [13] and [18], events with wrong-sign pion and $D$ meson charge combinarespectively. tions [14].

$D^{*+} \rightarrow \pi^{0} D^{+}$decay and one $\pi^{0}$, either from $D^{*+} \rightarrow D^{+} \pi^{0}$ or $\bar{D}^{* 0} \rightarrow \bar{D}^{0} \pi^{0}$ decay, to isolate the process and measure the $D^{*+} \bar{D}^{* 0}$ invariant mass [14]. The signal for real $\pi^{-} D^{*+} \bar{D}^{* 0}$ final states is the distinct peak near $2.15 \mathrm{GeV}$ in the mass distribution for the systems recoiling from the reconstructed $D^{+}$and $\pi^{-}$, shown in Fig. 6a. The measured $D^{*} \bar{D}^{*}$ invariant mass distribution for events in the $2.15 \mathrm{GeV}$ peak, inferred from the $\pi^{-}$momentum, is shown as the data points in Fig. $6 \mathrm{~b}$, where a strong near-threshold peaking behavior that cannot be described by a phase-space-like distribution, shown as a dash-dot blue curve, or by combinatoric background, which is determined from wrongsign (WS) events in the data (i.e., events where the pion and charged $D$ meson have the same sign) that are shown as the shaded histogram. The solid black curve shows the results of a fit to the data points that includes an efficiency weighted S-wave BW function (long dashes), the combinatoric background shape (short dashes) scaled to measured non- $D^{*+} \bar{D}^{* 0} \pi^{-}$background level under the signal peak in Fig. 6a, and a phase-space term (dash-dot). The fit returns a $13 \sigma$ signal with mass and width $M=4026.3 \pm 4.5 \mathrm{MeV}$ and $\Gamma=24.8 \pm 9.5 \mathrm{MeV}$, values that agree within errors to those measured for the $Z_{c}(4020)^{+} \rightarrow \pi^{+} \pi^{-} h_{c}$ channel. Although BESIII cautiously calls this $\left(D^{*} \bar{D}^{*}\right)^{+}$ signal the $Z_{c}(4025)$, this is most likely another decay mode of the $Z_{c}(4020)$.

A neutral $D^{*} \bar{D}^{*}$ state with a mass and width that are consistent with the $Z_{c}(4025)^{+}$was seen by BESIII in $e^{+} e^{-} \rightarrow \pi^{0}\left(D^{*} \bar{D}^{*}\right)^{0}$ events [19].

The $Z_{c}(4020) \rightarrow D^{*} \bar{D}^{*}$ and $\pi^{-} h_{c}$ signal yields reported in refs. [14] and [13], respectively, imply a partial width for $Z_{c}(4020) \rightarrow D^{*} \bar{D}^{*}$ that is larger than that for $Z_{c}(4020) \rightarrow \pi h_{c}$, but only by a factor of $12 \pm 5$, not by the large factors that are characteristic of conventional charmonium. In addition, there is no sign of $Z_{c}(4020) \rightarrow D \bar{D}^{*}$ in Fig. 4a, where there is a $\sim 500$ event $Z_{c}(3885) \rightarrow$ $D^{0} D^{*-}$ signal. A recent BESII study of $Z_{c}(3900) \rightarrow D \bar{D}^{*}$ decays [25] set a $90 \%$ confidence level upper limit $\mathscr{B}\left(Z_{c}(4020) \rightarrow D \bar{D}^{*}\right)<0.13 \times \mathscr{B}\left(Z_{c}(3885) \rightarrow D \bar{D}^{*}\right)$. This absence of any evident signal for $Z_{c}(4020) \rightarrow D \bar{D}^{*}$ means that the $Z_{c}(4020) \rightarrow D \bar{D}^{*}$ partial width is considerably smaller than that for $Z_{c}(4020) \rightarrow D^{*} \bar{D}^{*}$, which is suggestive of some relation to the $Z_{c}(4020)$ 's proximity to the $2 m_{D^{*}}$ threshold. 

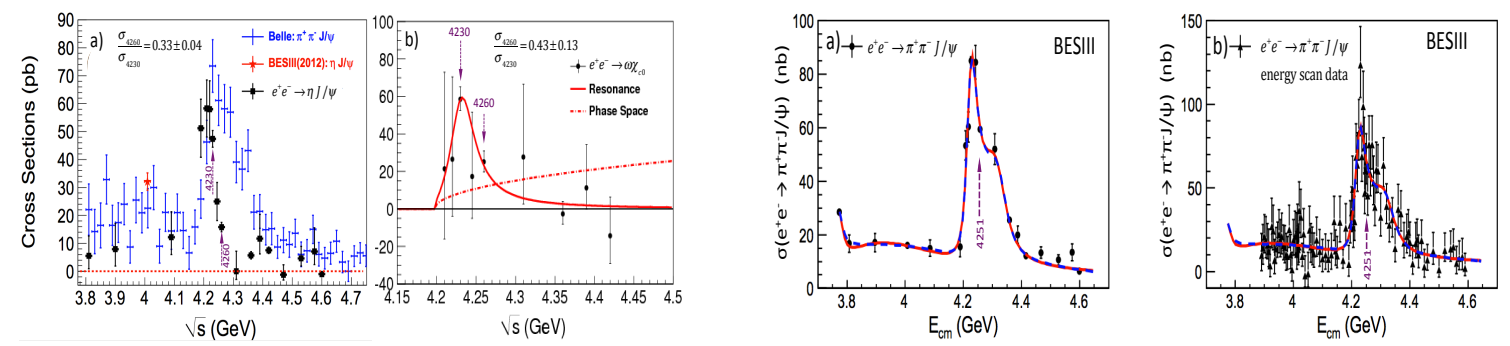

Figure 7: a) BESIII measurements of $\sigma\left(e^{+} e^{-} \rightarrow \eta J / \psi\right)$ Figure 8: BESIII measurements [23] of the cross section are shown as black points [20] and a red star [21]; Belle isr for $e^{+} e^{-} \rightarrow \pi^{+} \pi^{-} J / \psi$ for a) the "high luminosity" scan measurements of $\sigma\left(e^{+} e^{-} \rightarrow \pi^{+} \pi^{-} J / \psi\right)$ are shown as blue data and b) the "low luminosity" scan data. Dashed arcrosses [5]. b) Data points show BESIII $\sigma\left(e^{+} e^{-} \rightarrow \omega \chi_{c 0}\right)$ rows in both plots indicate the " $Y(4260)$ " mass value from measurements [22]. The solid (dash-dot) shows results of a the PDG-2016 average of results based on single-BW resofit of a threshold-constrained BW (phase-space-only) shape nance fits to pre-2016 measurements given in Eq. 2.1. to the data.

\subsubsection{BESIII discovery of a two-resonance $Y(4260)$ line-shape}

BESIII subsequently did a scan of measurements around the $E_{\mathrm{cm}}=4.26 \mathrm{GeV}$, including relatively high statistics points at $E_{\mathrm{cm}}=4230 \mathrm{MeV}, 4260 \mathrm{MeV}$ and $4360 \mathrm{MeV}^{3}$ With these data, BESIII measured the cross sections for $e^{+} e^{-} \rightarrow \eta J / \psi$ shown in Fig. 7a [20] and $e^{+} e^{-} \rightarrow \omega \chi_{c 0}$ shown in Fig. $7 b$ [22].

Figure 7a includes a comparison of BESIII's $e^{+} e^{-} \rightarrow \eta J / \psi$ cross sections with Belle isr results for $\sigma\left(e^{+} e^{-} \rightarrow \pi^{+} \pi^{-} J / \psi\right)$ [5], where it is evident that the peak seen in the $\eta J / \psi$ channel is much narrower than Belle's $Y(4260) \rightarrow \pi^{+} \pi^{-} J / \psi$ peak. The $\omega \chi_{c 0}$ cross section (Fig. 7b) shows a behavior that is similar as that for $\eta J / \psi$. The red curve in this figure is the result of a fit of a threshold-constrained BW resonance to the $\omega \chi_{c 0}$ data points, which returns mass and width values, $M_{\omega \chi_{c 0}}=4230 \pm 10 \mathrm{MeV}$ and $\Gamma_{\omega \chi_{c 0}}=38 \pm 12 \mathrm{MeV}$, that are a poor match the the PDG values for the $Y(4260)$ given in Eq. 2.1. The absence of any constraining $\eta J / \psi$ data points on the lower side of the peak, i.e., between $M(\eta J / \psi)=4100$ and $4200 \mathrm{MeV}$, prevented BESIII from doing a meaningful fit for a $\eta J / \psi$ line shape. Instead they characterized the shapes of the $\eta J / \psi$ and $\omega \chi_{c 0}$ peaks by the ratio of their cross sections at the high-statistics $E_{\mathrm{cm}}=4230$ and $4260 \mathrm{MeV}$ data points: $R_{4230}^{4260}(f)=\frac{\sigma^{4260}\left(e^{+} e^{-} \rightarrow f\right)}{\sigma^{4230}\left(e^{+} e^{-} \rightarrow f\right)}$, where they find good agreement: $R_{4230}^{4260}(\eta J / \psi)=0.33 \pm 0.04$ and $R_{4230}^{4260}\left(\omega \chi_{c 0}\right)=0.43 \pm 0.13$.

The evident incompatibility of the narrow structures in the $e^{+} e^{-} \rightarrow \eta J / \psi$ and $e^{+} e^{-} \rightarrow \omega \chi_{c 0}$ cross sections with the broad $Y(4260) \rightarrow \pi^{+} \pi^{-} J / \psi$ peak measured in isr production prompted BESIII to map out the $E_{\mathrm{cm}}$ energy region in the vicinity of the $Y(4260)$ with two additional, independent data sets [23]. One consists of 19 "high luminosity" data runs with at least $40 \mathrm{pb}^{-1} / \mathrm{point}$ between $E_{\mathrm{cm}}=3773 \mathrm{MeV}$ and $4599 \mathrm{MeV}$. The other consists of 60 "low luminosity" energy-scan data runs with $7-9 \mathrm{pb}^{-1} /$ point between $E_{\mathrm{cm}}=3882 \mathrm{MeV}$ and $4567 \mathrm{MeV}$. Figures $8 \mathrm{a}$ and b show $e^{+} e^{-} \rightarrow \pi^{+} \pi^{-} J / \psi$ cross section measurements from the high- and low-luminosity data scans, respectively, where it is evident that the line shape of the " $Y(4260)$ " peak is not well described by a single BW resonance function. The curves in the figures show the results from fits to the data in

\footnotetext{
${ }^{3}$ The three "high luminosity" data samples have integrated luminosities of $1047 \mathrm{pb}^{-1}$ at $4230 \mathrm{MeV}, 827 \mathrm{pb}^{=-1}$ at $4260 \mathrm{MeV}$; and $540 \mathrm{pb}^{-1}$ at $4360 \mathrm{MeV}$. The other points have luminosities of about $50 \mathrm{pb}^{-1}$.
} 
both plots with two interfering BW resonance amplitudes that provides mass and width values of

$$
\begin{array}{cl}
M_{1}=4220 \pm 4 \mathrm{MeV} & \Gamma_{1}=44 \pm 5 \mathrm{MeV} \\
M_{2}=4320 \pm 13 \mathrm{MeV} & \Gamma_{2}=101_{-22}^{+27} \mathrm{MeV}
\end{array}
$$

where the statistical and (smaller) systematic errors are added in quadrature. The simplest interpretation of these results is that the first peak is what we have been calling the $Y(4260)$, which now has a significantly lower mass and narrower width than the $B$-factory-measured values that are given above in Eq. 2.1, and that the second peak is the due to a $\pi^{+} \pi^{-} J / \psi$ decay mode of the $Y(4360)$ resonance, ${ }^{4}$ with slightly lower mass and narrower width values than those determined from its $\pi^{+} \pi^{-} \psi^{\prime}$ decay mode (as listed in PDG-2016 tables [6]).

BESIII measurements of the energy dependence of the cross section for $e^{+} e^{-} \rightarrow \pi^{+} \pi^{-} h_{c}$ with the same two data sets [26] are shown in Fig. 9. The solid red curve in the figure shows the results of a fit to the measurements with a coherent sum of two BW amplitudes. The parameters determined from the fit are:

$$
\begin{array}{ll}
M_{1}=4218 \pm 4 \mathrm{MeV} & \Gamma_{1}=66 \pm 9 \mathrm{MeV} \\
M_{2}=4392 \pm 6 \mathrm{MeV} & \Gamma_{2}=140 \pm 16 \mathrm{MeV}
\end{array}
$$

where the statistical and (smaller) systematic errors are added in quadrature. The lower mass BW term, shown in the figure as a dashed green line, has a fitted mass and width that are consistent with the $M \simeq 4220 \mathrm{MeV}$ peak seen in $\eta J / \psi, \omega \chi_{c 0}$ and $\pi^{+} \pi^{-} J / \psi$. No evidence for the higher mass $\pi^{+} \pi^{-} h_{c}$ peak is seen in the $\eta J / \psi$ or $\omega \chi_{c 0}$ channels and its measured parameters are inconsistent with those of the $Y(4320)$, for both the $\pi^{+} \pi^{-} J / \psi$ and $\pi^{+} \pi^{-} \psi^{\prime}$ channels.
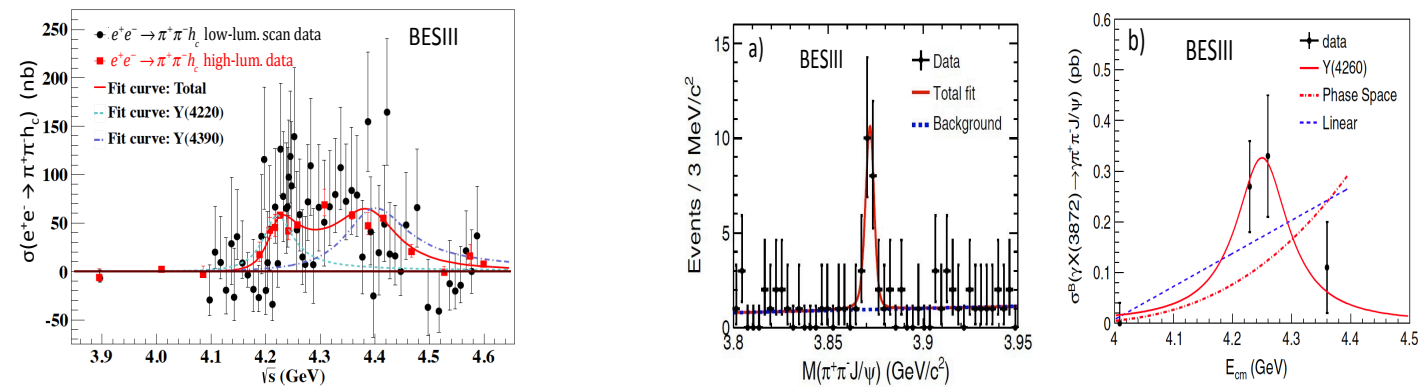

Figure 9: The data points show BESIII measure- Figure 10: a) The data points show the $M\left(\pi^{+} \pi^{-} J / \psi\right)$ disments of $\sigma\left(e^{+} e^{-} \rightarrow \pi^{+} \pi^{-} h_{c}\right)$, where the $h_{c}$ was tribution for $e^{+} e^{-} \rightarrow \gamma \pi^{+} \pi^{-} J / \psi$ events at energies near the detected via its $h_{c} \rightarrow \gamma \eta_{c}$ decay mode with the $\eta_{c} Y(4260)$ [27]. The fitted mass and width, $M=3871.9 \pm$ reconstructed in one of 16 exclusive multihadron $0.7 \mathrm{MeV}$ and $\Gamma=0.0_{-0.0}^{+1.7} \mathrm{MeV}(<2.4 \mathrm{MeV})$, agree well decay channels [26]. The solid black dots are from with the PDG world average values for the $X(3872)$. b) The the low-luminosity-scan and the solid red points are energy dependence of the BESIII $\sigma\left(e^{+} e^{-} \rightarrow \gamma X(3872)\right) \times$ from the high-luminosity-scan. The solid red curve $\mathscr{B}\left(X(3872) \rightarrow \pi^{+} \pi^{-} J / \psi\right)$ measurements. The solid curve is shows results of a fit to the data with a coherent sum the $Y(4260)$ line shape fitted to the data; the dashed curves show of two interfering BW amplitudes. phase-space and linear production model expectations.

${ }^{4}$ The $Y(4360)$ is a $\pi^{+} \pi^{-} \psi^{\prime}$ resonance that was discovered by BaBar in the isr process $e^{+} e^{-} \rightarrow \gamma_{\text {isr }} \pi^{+} \pi^{-} \psi^{\prime}$ [24]. The PDG-2016 mass and width values are $4361 \pm 13 \mathrm{MeV}$ and $74 \pm 18 \mathrm{MeV}$, respectively [6]. 


\subsubsection{A connection between the $Y(4260)$ and the $X(3872)$ ?}

Another intriguing aspect of the $Y(4260)$ is the BESIII experiment's report of a strong signal for $X(3872)$ production in the process $e^{+} e^{-} \rightarrow \gamma \pi^{+} \pi^{-} J / \psi$ at c.m. energies in the region of the $Y(4260)$ resonance peak [27]. The $X(3872)$ was detected via its $\pi^{+} \pi^{-} J / \psi$ decay channel; a $\pi^{+} \pi^{-} J / \psi$ invariant mass distribution summed over the data at four energy points is shown in Fig. 10a, where a peak (with a significance of $6.3 \sigma$ ) at the mass of the $X(3872)$ is evident. Figure 10b shows the energy dependence of the $X(3872)$ production rate where there is some indication that the observed signal is associated with the $Y(4260)$. Assuming that $Y(4260) \rightarrow \gamma X(3872)$ decays are the source of this signal, and using the PDG lower limit $\mathscr{B}\left(X(3872) \rightarrow \pi^{+} \pi^{-} J / \psi\right)>$ 0.026 [6], BESIII determines

$$
\frac{\mathscr{B}(Y(4260) \rightarrow \gamma X(3872))}{\mathscr{B}\left(Y(4260) \rightarrow \pi^{+} \pi^{-} J / \psi\right)}>0.05,
$$

which is substantial and suggests that there is some commonality in the nature of the $Y(4260)$, $X(3872), Z_{c}(3900)$ and $Z_{c}(4020)$.

\section{Theoretical Models Proposed for the $\mathrm{Y}(\mathbf{4 2 6 0})$}

Three different theoretical points of view have been advocated as explanations for the existence and properties of the $Y(4260)$ :

Molecular models in which a color-singlet $D$ and anti- $D_{1}(2420)$ meson ${ }^{5}$ in an S-wave attract each other by Yukawa-like $\pi$ - and other light meson exchanges and bind in a manner similar to the way a neutron and proton bind to form a deuteron (Fig. 11a) [28]. For molecular states, the binding energy is not expected to be large and the bound state should have a mass that is near the sum of the masses of the constituent particles. ${ }^{6}$ Also the width cannot be narrower than that of its widest constituent [29].

QCD tetraquark models in which an (anti-colored) diquark comprised of charmed quark and a light quark, $[c q]$, binds via gluon exchange with a (colored) diantiquark that is comprised of an anti-charmed quark and a light antiquark, $[\bar{c} \bar{q}]$, to form a tightly bound four-quark state (Fig. 11b) [30]. Since the binding is due to the very strong color force, QCD tetraquarks can be tightly bound and, since in that case there would be considerable overlap of the constituent quarks and antiquarks, are expected to be very short-lived, i.e. wide.

QCD hybrids in which a colored charmed- and anticharmed-quark pair combine with a colored "valence" gluon to form a non-charmonium meson (Fig. 11c) [31]. The preferred decay modes are meson with its quarks in an S-wave plus an anti-meson with quarks in a P-wave (or vice-vers $a^{7}$ ) [32]. For the $Y(4260)$ the only accessible final-state that satisfies this condition would be $D^{*} \bar{D}_{0}^{*}(2400)$, where the $D_{0}^{*}(2400)$ is a broad $D \pi$ scalar resonance with mass $\simeq$ $2318 \mathrm{MeV}$ and width $\simeq 267 \mathrm{MeV}$. Lattice QCD calculations indicate that the lightest $J^{P C}=$ $1^{--} c \bar{c}$-gluon hybrid mass is about $4285 \mathrm{MeV}$ [33].

\footnotetext{
${ }^{5}$ The $D_{1}(2420)$ is an open-charm axial-vector $D^{*} \pi$ resonance with mass $2421 \mathrm{MeV}$ and width $27.4 \mathrm{MeV}$.

${ }^{6}$ Typical binding energies per nucleon in nuclei are $8 \sim 10 \mathrm{MeV}$.

${ }^{7}$ In this report inclusion of the charge conjugate combination is always implied.
} 
Hadrocharmonium is a model in which the strong partial width for the $Y(4260)$ meson to decay to $\pi^{+} \pi^{-} J / \psi\left(\pi^{+} \pi^{-} \psi^{\prime}\right)$, an OZI-suppressed process for charmonium, is explained by a meson structure that contains a color-singlet charmonium-state core surrounded by a light quark excitation (Fig. 11d) [34]. In the case of the $Y$ (4260), this core state was taken to be the $J / \psi$. Since the $J / \psi$ state is present in its constituents, the $Y(4260)$ naturally prefers to decay to final states that include it.

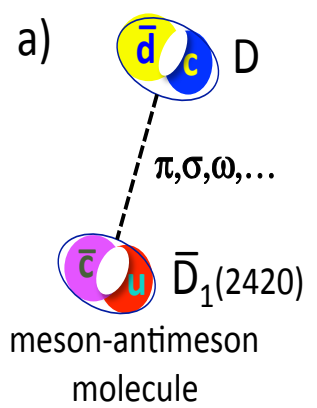

b)

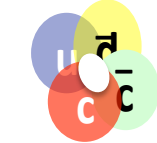

tetraquarks c)

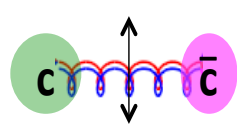

hybrids d)

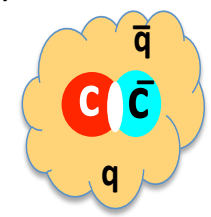

hadrocharmonium

Figure 11: Models proposed for the $Y(4260)$ : a) a bound $D-D_{1}(2460)$ molecule; b) a $[c q]-[\bar{c} \bar{q}]$ tetraquark; c) a $c \bar{c}$-gluon hybrid; d) hadrocharmonium.

Most of the theoretical discussions to date have been focused on $Y(4260)$ mass and width parameters that were determined from single BW fits to isr line shapes shown in Fig. 1. However, the recent measurements of the $e^{+} e^{-} \rightarrow \eta J / \psi, \omega \chi_{c 0}$ and $\pi^{+} \pi^{-} h_{c}$ cross sections, shown in Figs. 7 and 9, respectively, and precise results for $e^{+} e^{-} \rightarrow \pi^{+} \pi^{-} J / \psi$ shown in Fig. 8 demonstrate that the single-resonance assumptions that were used to determine the mass and width values given in Eq. 2.1 were too naïve and the values that were derived were not reliable. As a result, theoretical papers that interpreted the $Y(4260)$ as a bound state of a $D$ meson and a $\bar{D}_{1}(2420)[35,36]$, originally had to deal with a $D \bar{D}_{1}$ binding energy of $\simeq 35 \mathrm{MeV}$, which is quite a bit larger than typical values for nuclear systems bound by Yukawa-like meson-exchange forces. Now, with the lower Eq. 2.2 value for the $Y(4260)$ mass, the implied $D \bar{D}_{1}$ binding energy nearly doubles to $66 \mathrm{MeV}$, which makes the $D \bar{D}_{1}$ molecule interpretation pretty implausible. The lower mass and an asymmetric line shape are anticipated by the molecular model of ref. [37], which was based on fits to BESIII $\pi D \bar{D}^{*}$ [12] and Belle $\pi^{+} \pi^{-} J / \psi$ [5] data that were available at that time. However, this paper provides no dynamical explanation for such a large binding energy.

Other authors have suggested that the $Y(4260)$ might be a $c \bar{c}$-gluon hybrid meson [38, 39, 40]. A lattice QCD calculation (with pion mass $\sim 400 \mathrm{MeV}$ ) finds a candidate for a $1^{--}$hybrid state at a mass of $4285 \pm 14 \mathrm{MeV}$ that the authors suggested as a possible interpretation for the $Y(4260)$ based on the previous, Eq. 2.1 mass value measurement [33]. However, the new BESIII mass measurement establishes an even larger discrepancy with the LQCD prediction and weakens the case for this assignment.

In the QCD tetraquark model, the $Y(4260)$ and $X(3872)$ are symmetric $[c q]_{S=0}[\bar{c} \bar{q}]_{S=1}$ diquarkdiantiquark combinations. The difference between them is that in the $X(3872)$, the $[c q]$ and $[\bar{c} \bar{q}]$ are in a relative S-wave, while in the $Y(4260)$, they are in a relative $\mathrm{P}$-wave [41]. The $\simeq 350 \mathrm{MeV}$ mass difference between the two states is consistent with the typical mass penalty that is associated 
with the addition of one unit of orbital angular momentum. In this scheme, the strong $Y(4260) \rightarrow$ $\gamma X(3872)$ transition reported by BESIII [27] is an allowed electric dipole transition between the two related states [42]. Likewise the $Z_{c}(3900)$ can be naturally accommodated as the antisymmetric, Swave $[c q]_{S=0}\left[\bar{c} \bar{q}^{\prime}\right]_{S=1}$ diquark-diantiquark combination, and the $Z(4430)$ as its first radial excitation. A problem with the diquark picture is that, since the strong radial color force between the diquark and the diantiquark is universal, it has the same strength for $q, q^{\prime}=u, d, s$ independently of their flavor or spin state. As a result, every successful application of the idea to an experimentally observed state carries with it predictions for a large number of related states that, in most cases, have not been seen.

The hadrocharmonium model has problems with the BESIII discovery of the two-resonance structure of the $Y(4260)$, in particular with the observations that both of the component resonances are seen to decay to $\pi^{+} \pi^{-} J / \psi$ [23], while only the lower-mass one is seen to decay to $\pi^{+} \pi^{-} h_{c}$ [26]. In the $J / \psi$, the $c$ - and $\bar{c}$-quarks are in a spin-triplet state, while in the $h_{c}$ they are in a spin-singlet. By themselves, the triplet and singlet cores cannot mix and a hadrocharmonium state should have strong rates for decays to one of them, but not both. One way around this would be a model where there are two hadrocharmonium states, one with a spin-triplet core and the other with a spin-singlet core and these two states mix, producing the two observed resonances that comprise the $Y(4260)$ peak [43]. In this case both can decay to singlet and triplet states with relative rates determined by the (unspecified) mixing angle. However, in this scenario, it is still not possible to explain the observed pattern where the lower-mass state decays to both $\pi^{+} \pi^{-} J / \psi$ and $\pi^{+} \pi^{-} h_{c}$ while the higher-mass decays to $\pi^{+} \pi^{-} J / \psi$, but (apparently) not to $\pi^{+} \pi^{-} h_{c}$.

\section{Probing the $\mathrm{Y}(\mathbf{4 2 6 0})$ with $\pi^{-}\left(\mathrm{DD}^{*}\right)^{+}$Decays}

A promising future direction of $Y(4260)$-related research at BESIII will involve the use of high-statistics samples of $Y(4260) \rightarrow \pi^{-}\left(D \bar{D}^{*}\right)^{+}$to probe the underlying nature of the resonance. As shown above in Figs. 4 and 6, these are very prominent decay modes with a rich final-state structure. These decays are potentially powerful probes of the internal structure of the $Y(4260)$. For example, if the $Y(4260)$ is a tightly bound $D \bar{D}_{1}(2420)$ molecule as suggested be ref. [37], this should show up as distinct $M\left(D^{*} \pi\right)$ features in the $Y(4260) \rightarrow \pi D \bar{D}^{*}$ Dalitz plot. On the other hand, if the $Y(4260)$ is a QCD hybrid, the non- $Z_{c}(3900)$ contributions to $\pi D \bar{D}^{*}$ final states should have a strong $D^{*} \bar{D}_{0}^{*}(2400)$ component that would contribute a non-resonant-like $D \pi$ component that peaks at the upper edge of the $M^{2}(D \pi)$ axis of the Dalitz plot.

\subsection{Experimental considerations}

Unpublished BESIII measurements of the $E_{\mathrm{cm}}$-dependence of $\sigma\left(e^{+} e^{-} \rightarrow \pi^{+} D^{0} D^{*-}\right)$ are shown in Fig. 12 [44]. There, two striking structures are evident: one is a peak at $4225 \pm 6 \mathrm{MeV}$ that is $72 \pm 9 \mathrm{MeV}$ wide; the second is a peak at $4400 \pm 9 \mathrm{MeV}$ that is $182 \pm 17 \mathrm{MeV}$ wide. The low-mass peak is reasonably consistent with the low-mass peak seen in $\eta J / \psi, \omega \chi_{c 0}, \pi^{+} \pi^{-} J / \psi$ and $\pi^{+} \pi^{-} h_{c}$ (as discussed above) and the second peak is consistent with the second peak seen in $\pi^{+} \pi^{-} h_{c}$. There is no hint of a signal for for the $M=4320 \mathrm{MeV}$ peak seen in the $Y(4260) \rightarrow \pi^{+} \pi^{-} J / \psi$ (i.e., in Fig. 8). This suggests that an amplitude analysis of $Y(4260) \rightarrow \pi D \bar{D}^{*}$ should concentrate on data near $E_{\mathrm{cm}}=4.22 \mathrm{GeV}$, i.e., near the peak of the lower-mass resonance. 

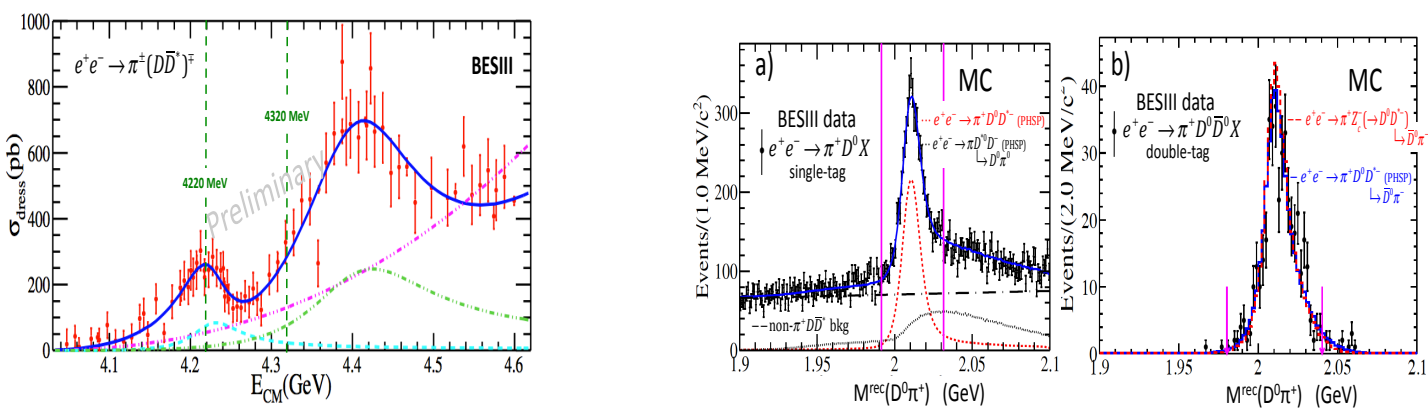

Figure 12: Preliminary BESIII measurements [44]) Figure 13: a) The $M^{\mathrm{rec}}\left(D^{0} \pi^{+}\right)$distribution for single-tag of $\sigma\left(e^{+} e^{-} \rightarrow \pi^{+} D^{0} D^{*-}\right)$ for $4.0 \leq E_{\mathrm{cm}} \leq 4.6 \mathrm{GeV}$. $e^{+} e^{-} \rightarrow \pi^{+} D^{0}$ events at $E_{\mathrm{cm}}=4.6 \mathrm{GeV}$ from BESIII [44]. The solid blue curve shows results of a fit that in- The curves are described in the text. b) The $M^{\text {rec }}\left(D^{0} \pi^{+}\right)$ cludes a coherent sum of two BW resonance terms distribution for double-tag $e^{+} e^{-} \rightarrow \pi^{+} D^{0} \bar{D}^{0} X$ events, where (dash and dash-2dots) and non-resonant phase-space $M(X)=m_{\pi} \pm 30 \mathrm{MeV}$ [25]. (dash-3dots).

The BESIII efficiencies for fully reconstructing $e^{+} e^{-} \rightarrow \pi^{+} D \bar{D}^{*}$ decays at $E_{\mathrm{cm}} \simeq 4.22 \mathrm{GeV}$ are pitifully small. This is because the $\bar{D}^{*}$-mesons are nearly at rest and charged $\pi^{-}$-mesons from $D^{*-} \rightarrow D^{0} \pi^{-}$decays have very low momenta, $\simeq 40 \mathrm{MeV} / c$, and, at best, produce only partial tracks in the charged particle tracking systems, and the $\gamma$-rays from $D^{*} \rightarrow D \pi^{0} ; \pi^{0} \rightarrow \gamma \gamma$ are below $100 \mathrm{MeV}$ and are measured with low efficiency and poor energy resolution. The useful decay modes for reconstructing $D^{0}$ and $D^{-}$mesons have branching fractions that are generally less than $10 \%$ and, since there are two $D$-mesons in the final state, the useful product branching fractions are a few percent and, when experimental efficiencies are included, the overall efficiencies drop to a few tenths of a percent. Thus, BESIII measurements of $\pi^{+} D \bar{D}^{*}$ rely heavily on partial reconstruction techniques.

The simplest and most efficient partial reconstruction technique is the "single-tag" method in which only the ("bachelor") $\pi^{+}$and one of the final-state $D$ mesons are reconstructed, and the presence of the undetected $D^{*}$ is inferred from the mass recoiling against the detected $\pi^{+} D$ system:

$$
M^{\mathrm{rec}}\left(D \pi^{+}\right)=\sqrt{\left(E_{\mathrm{cm}}-E_{\pi^{+}}-E_{D}\right)^{2}-\left|\vec{p}_{\pi^{+}}+\vec{p}_{D}\right|^{2}} .
$$

The results are illustrated in Fig. 13a that shows the $M^{\mathrm{rec}}\left(D^{0} \pi^{+}\right)$distribution measured by BESIII at $E_{\mathrm{cm}}=4.260 \mathrm{eV}$ [44]. Here the striking peak near $M^{\mathrm{rec}}\left(D^{0} \pi^{+}\right)=2.01 \mathrm{GeV}$ is a signal for the reaction $e^{+} e^{-} \rightarrow \pi^{+} D^{0} D^{*-}$, where the $D^{*-}$ is undetected. In addition there is non-peaking background from non- $\pi^{+} D \bar{D}^{*}$ processes and feedthrough from the isospin partner reaction $\pi^{+} D^{* 0} D^{-}$, where the detected $D^{0}$ originates from $D^{* 0} \rightarrow D^{0} \pi^{0}$ or $D^{0} \gamma$. The three components of the distribution have different shapes and can be identified on a statistical basis from fits to the $M^{\text {rec }}$ distribution as illustrated in the figure. This method was used to measure the $e^{+} e^{-} \rightarrow \pi^{+} D^{0} D^{*-}$ cross sections shown in Fig. 8.

The single-tag method has substantial background, does not provide information about the decay of the $\bar{D}^{*}$ and cannot determine on an event-by-event basis if the detected $D$ or the undetected $\bar{D}$ is the one that originates from a $D^{*}$. This can be improved upon, albeit with an order-of-magnitude sacrifice in event rate, by reconstructing both the $D$ and the $\bar{D}$ (in addition to the bachelor pion) in the event and requiring the undetected recoil mass to be consistent with $m_{\pi}$. The $M^{\text {rec }}\left(D^{0} \pi^{+}\right)$distri- 
bution for reconstructed $\left.e^{+} e^{-} \rightarrow \pi^{+} D^{0} \bar{D}^{0}(\pi)\right)$ events at $E_{\mathrm{cm}}=4.23 \mathrm{GeV}$ from BESIII is shown in Fig. 13b, where a clear signal for $D^{*-} \rightarrow \bar{D}^{0} \pi^{-}$, with no significant background, can be seen [25]. Here the low momentum $\pi^{-}$from $D^{*-} \rightarrow \bar{D}^{0} \pi^{-}$is not reconstructed, but it is known to be a pion from the measured value of $M^{\mathrm{rec}}\left(\pi^{+} D^{0} \bar{D}^{0}\right)$; charge conservation requires it to be negatively charged; its negative charge and the $M^{\mathrm{rec}}\left(D^{0} \pi^{+}\right)$indicates that it originated from $D^{*-} \rightarrow \bar{D}^{0} \pi^{-}$ decay; and its c.m. momentum is $\vec{p}_{\pi^{-}}=-\left(\vec{p}_{\pi}^{+}+\vec{p}_{D^{0}}+\vec{p}_{\bar{D}^{0}}\right)$. Thus, in this particular "double-tag" event sample, the background is negligible, the four-vectors of all of the final state particles are measured, and the assignment of which tracks form the $D^{*}$ is unambiguous. (This latter point is not the case for all double-tag sample. Events in which the reconstructed particles are $\pi^{+} D^{0} D^{-}$ and the missing mass is consistent with $m_{\pi}$ could originate from either $\pi^{+} D^{0} D^{*-}$ or $\pi^{+} D^{* 0} D^{-}$.)

\subsection{Testing the $\mathrm{Y}(4260)$ is a $\mathrm{D} \bar{D}_{1}(2420)$ molecule conjecture}

Completely reconstructed $Y(4260) \rightarrow \pi^{+} D^{0} D^{*-}$ decays can provide a powerful test of the hypothesis that the $Y(4260)$ is a $D \bar{D}_{1}(2420)$ molecule, as suggested in ref. [37]. The pion angular distribution for $e^{+} e^{-} \rightarrow \pi D \bar{D}^{*}$ where the $D \bar{D}^{*}$ originates from $Z_{c}(3885)$ decay, shown in Fig. $4 \mathrm{~b}$, is consistent with being flat. This was interpreted by the BESIII group as an indication that the process is dominated by an $\mathrm{S}$-wave and used this to claim a $J^{P}=1^{+}$assignment for the $Z_{c}(3885)$. However in the $D \bar{D}_{1}$ molecule model for ref. [37], $Z_{c}$ production occurs via the $D_{1}-D^{*}-\bar{D}$ triangle diagram shown in Fig. 14 a where the $D_{1}$ is virtual. In this case, the pion is produced at the $D_{1} \rightarrow D^{*} \pi$ vertex, which is well known to be dominated by a $D$-wave final state, a behavior that was predicted by Isgur and Wise based on heavy quark symmetry [45], and confirmed experimentally by CLEO [46] and Belle [47]. Belle data on $B \rightarrow D_{1}(2420) \pi$ are shown if Fig. 15, where the influence of the strong D-wave in $D_{1}(2420) \rightarrow D^{*} \pi$ is apparent. Thus, if the $D_{1}(2420) \rightarrow D^{*} \pi$ vertex is playing a major role in $Y(4260) \rightarrow \pi D \bar{D}^{*}$ decays, its dominant $\mathrm{D}$-wave behavior must somehow be masquerading as the flat, apparently S-wave, production distribution seen in Fig. $4 \mathrm{~b}$.
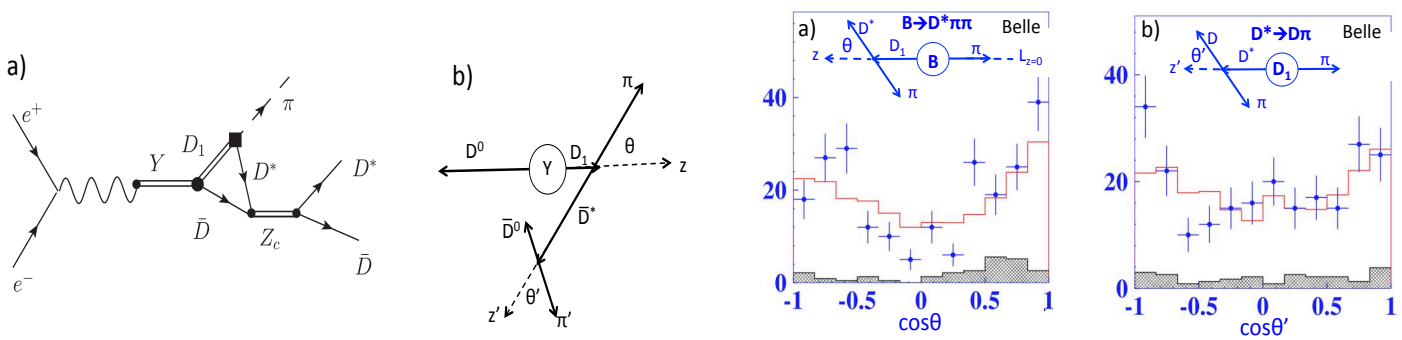

Figure 14: a) The $Y(4260) \rightarrow \pi Z_{c}(3900)$ decay process Figure 15: The a) bachelor-pion and b) decaymediated by a $D_{1}(2420) \bar{D}$ loop in the molecule model of pion helicity-angle distributions $B \rightarrow D_{1}(2420) \bar{D}$ deref. [37]. In the text, the $\pi$-meson from the $D_{1} \rightarrow D^{*} \pi$ cays from Belle [47]. Here, since both the parent Bvertex is referred to as the "bachelor" pion. b) The defi- meson and the recoiling pion has zero spin, the $D_{1}$ nitions of the $\theta$ and $\theta^{\prime}$ helicity angles. The $\pi$-meson from mesons are aligned in a $|j m\rangle=|10\rangle$ state, where the the $D^{*} \rightarrow D \pi$ vertex is referred to as the "decay" pion. $\quad z$-axis is the direction of the $\bar{D}$ in the $\pi D^{*}$ rest frame.

It turns out that this masquerading is not so difficult, although it requires fine tuning. For $e^{+} e^{-} \rightarrow \pi D \bar{D}^{*}$ production via a $D \bar{D}_{1}$ intermediate state, the projected one-dimensional bachelor 
pion angular distribution is expected to be ${ }^{8}$

$$
\frac{d N}{d \cos \theta} \propto 2 r+\frac{1-r}{2}\left(5-3 \cos ^{2} \theta\right)-\sqrt{2 r(1-r)} \cos \phi\left(1-3 \cos ^{2} \theta\right),
$$

where $\theta$ is the $D_{1} \rightarrow \pi D^{*}$ helicity angle (see Fig. 14b), $r=\Gamma_{S} /\left(\Gamma_{S}+\Gamma_{D}\right)$ is the relative strength of the $\mathrm{S}$-wave contribution, and $\phi$ is the relative phase between the $\mathrm{S}$ - and $\mathrm{D}$-wave amplitudes. Here it is easy to show that the angular distribution is flat if $r=1 /\left(1+8 \cos ^{2} \phi\right)$. So, if $\cos ^{2} \phi \approx 1$, a $\simeq 10 \% \mathrm{~S}$-wave contribution would suffice. With a $\pi^{+} D^{0} \bar{D}^{0}$ double-tag data sample, the $D^{*} \rightarrow D \pi$ helicity angle distribution could also be studied. In this case the projected one-dimensional decay pion angular distribution behaves as

$$
\frac{d N}{d \cos \theta^{\prime}} \propto 2 r+\frac{1-r}{2}\left(5-3 \cos ^{2} \theta^{\prime}\right)-\sqrt{2 r(1-r)} \cos \phi\left(1-3 \cos ^{2} \theta^{\prime}\right)
$$

In this case, the same value of $r$, i.e. $r=1 /(1+8 \cos \phi)$ would produce a flat distribution. Thus, even if the measured one-dimensional distributions show flat $\cos \theta$ and $\cos \theta^{\prime}$ behaviors, these would not unambiguously rule out a dominant D-wave contribution as expected for the $D \bar{D}_{1}(2420)$ molecular picture.

The best hope for a definitive test would be a full, three-dimensional analysis that simultaneously fits to $\cos \theta, \cos \theta^{\prime}$ and $\chi$, where $\chi$ is the angle between the $D_{1} \rightarrow D^{*} \pi$ and $D^{*} \rightarrow D \pi$ decay planes. For the production-decay chain $e^{+} e^{-} \rightarrow D \bar{D}_{1} ; \bar{D}_{1} \rightarrow \bar{D}^{*} \pi ; \bar{D}^{*} \rightarrow \bar{D} \pi$, this distribution, in terms of helicity amplitudes $A_{10}$ and $A_{00}$, is

$$
\begin{aligned}
\frac{d^{3} N}{d \cos \theta d \cos \theta^{\prime} d \chi} & =\left|A_{10}\right|^{2} \frac{\sin ^{2} \theta^{\prime}}{4}\left(1+\cos ^{2} \theta\right)+\left|A_{10}\right|^{2} \frac{\cos ^{2} \theta^{\prime}}{2}\left(1-\cos ^{2} \theta\right) \\
& +\frac{1}{4}\left|A_{10}\right|^{2} \sin ^{2} \theta \sin ^{2} \theta^{\prime} \cos (2 \chi)+\mathscr{R}\left(A_{10}^{*} A_{00}\right) \sin \theta \cos \theta \sin \theta^{\prime} \cos \theta^{\prime} \cos \chi
\end{aligned}
$$

where $A_{10}$ and $A_{00}$ are related to the S- and D-wave partial wave amplitudes by

$$
\begin{aligned}
& A_{10}=\frac{S}{\sqrt{3}}+\frac{D}{\sqrt{6}} \\
& A_{00}=\frac{S}{\sqrt{3}}-\sqrt{\frac{2}{3}} D .
\end{aligned}
$$

The dominance of the D-wave amplitude in $\bar{D}_{1}(2420) \rightarrow D^{*-} \pi^{+}$vertex provides a strong signature for testing the molecule picture for the $Y(4260)$. If three-dimensional fits to the $Y(4260) \rightarrow$ $\pi^{+} D^{0} D^{*-}$ return a value for the $\mathrm{S}$-wave amplitude that is unambiguously comparable to, or larger than, that for the D-wave, the $D \bar{D}_{1}(2420)$ model can be excluded. In addition, in the molecule picture the $D_{1}(2420)$ is off-shell and the $D^{*} \pi$ invariant mass distribution must be modulated by a propagator that favors large $D^{*} \pi$ invariant mass values. Among other things, this would produce a distinct excess of events with small pion helicity angle values in the $D \pi$ rest frame.

\footnotetext{
${ }^{8}$ Since the $D \bar{D}_{1}$ system is produced by a virtual photon and the $D$-meson has zero spin, the $D_{1}$ mesons are aligned with $|j m\rangle=|1 \pm 1\rangle$, where the $z$-axis is along the beam line.
} 


\subsection{Testing the QCD tetraquark model for the $\mathrm{Y}(\mathbf{4 2 6 0})$}

On the other hand, in the QCD tetraquark picture, where the $Y(4260)$ is a $[c q]_{S=0}$ diquark that is bound, in a P-wave, to a $[\bar{c} \bar{q}]_{S=1}$ diantiquark by the color force, and the $Z_{c}(3900)$ is a similar structure in an S-wave, the decay $Y(4260) \rightarrow \pi D \bar{D}^{*}$ is unrelated to $D \bar{D}_{1}$ production. In this case, the decay goes directly via $Y(4260) \rightarrow \pi Z_{c}(3900)$, for which studies of $Z_{c}(3900)$ production in $\pi^{+} \pi^{-} J / \psi$ final states find a D-wave contribution that is negligibly small [15]. In this case, the relevant helicity amplitude relation governing the $\pi D \bar{D}^{*}$ final state has the same form as Eq. 4.5 but with the interchange of the bachelor pion and the $D^{0}$ (i.e., where $\theta$ is taken to be the helicity angle of the $D^{0}$ meson in $Z_{c}(3900) \rightarrow D^{0} D^{*-}$ rest frame).

\subsection{Testing the cê-gluon hybrid model for the $\mathrm{Y}(\mathbf{4 2 6 0})$}

In the QCD-hybrid model for the $Y(4260)$, the $Y(4260) \rightarrow \pi^{+} D^{0} D^{*-}$ decay process should be dominated by $e^{+} e^{-} \rightarrow D_{0}^{*}(2400)^{+} D^{*-}$ with the $D_{0}^{*}$ and $D^{*-}$ in an S-wave followed by $D_{0}^{*}(2400)^{+} \rightarrow$ $D^{0} \pi^{+}$decay, also in an S-wave. In this case, the final state angular momentum is all carried by the $D^{*-}$ in an aligned $|j m\rangle=|1 \pm 1\rangle$ angular momentum state. In this case, the $\cos \theta$ distribution would be flat but the $\cos \theta^{\prime}$ distribution (for $D^{*-} \rightarrow \bar{D}^{0} \pi^{-}$) distribution would be quite dramatic:

$$
\frac{d N}{d \cos \theta^{\prime}} \propto \frac{3}{4} \sin ^{2} \theta^{\prime}
$$

\section{Discussion}

The BESIII double-tag $e^{+} e^{-} \rightarrow \pi D \bar{D}^{*}$ analysis [25] found $384 \pi^{ \pm} D^{0} \bar{D}^{0}$ events in the existing, $1092 \mathrm{pb}^{-1}$ data sample collected at $4230 \mathrm{MeV}$, near the peak of the $e^{+} e^{-} \rightarrow Y(4260) \rightarrow \pi D \bar{D}^{*}$ cross section and $207 \pi^{ \pm} D^{0} \bar{D}^{0}$ events at $4260 \mathrm{MeV}$, which, according to Fig. 12, are predominantly from the non-resonant background. Given their low background, as is evident in Fig. 13b, the 384 event sample at the $Y(4260)$ should be enough to support a conclusive three-dimensional analysis if one of the three previously mentioned scenarios is dominant. ${ }^{9}$ Ultimately, analyses that include and measure contributions from all possible sources will be necessary. This will require much more BESIII data, which is expected in the future. The work described here should be considered as a first step in a long, but interesting and, hopefully, informative process.

\section{Acknowledgements}

I congratulate the Bormio 2017 organizers for once again arranging an interesting and successful meeting, and I thank them for inviting me to give this report. This work was supported by the Institute for Basic Science (Korea) under project code: IBS-R016-D1.

\footnotetext{
${ }^{9} \mathrm{~A}$ four-dimensional Belle analysis of a $2000 B \rightarrow K^{-} \pi^{+} \psi^{\prime}$ events produced conclusive results on the existence and properties of the $Z(4430)^{+} \rightarrow \pi^{+} \psi^{\prime}$ resonance even though the $Z(4430)$ contribution to the event sample was only at the $10 \%$ level [48].
} 


\section{References}

[1] B. Aubert et al. (BaBar Collaboration), Phys. Rev. Lett. 95, 142001 (2005).

[2] J.P. Lees et al. (BaBar Collaboration), Phys. Rev. D86, 051102 (2012).

[3] T.E. Coan et al. (CLEO Collaboration), Phys. Rev. Lett. 96, 162003 (2006).

[4] C.Z, Yuan et al. (Belle Collaboration), Phys. Rev. Lett. 99, 182004 (2007).

[5] Z.Q. Liu et al. (Belle Collaboration), Phys. Rev. Lett. 110, 252002 (2013).

[6] C. Patrignani et al. (Particle Data Group), Chin. Phys. C40, 100001 (2016).

[7] J.Z. Bai et al. (BES Collaboration), Phys. Rev. Lett. 88, 101802 (2001).

[8] J.Z. Bai et al. (BES Collaboration), Phys. Rev. Lett. 84, 594 (1999).

[9] M. Ablikim et al. (BES Collaboration), Phys. Lett. B660, 315 (2008).

[10] N. Brambilla et al., Eur. Phys. J. C71, 1534 (2011).

[11] M. Ablikim et al. (BESIII Collaboration), Phys. Rev. Lett. 110, 252001 (2013).

[12] M. Ablikim et al. (BESIII Collaboration), Phys. Rev. Lett. 112, 022001 (2014).

[13] M. Ablikim et al. (BESIII Collaboration), Phys. Rev. Lett. 111, 242001 (2013).

[14] M. Ablikim et al. (BESIII Collaboration), Phys. Rev. Lett. 112, 132001 (2014).

[15] M. Ablikim et al. (BESIII Collaboration), submitted for publication in Phys. Rev. Lett.

[16] M. Ablikim et al. (BESIII Collaboration), Phys. Rev. Lett. 115, 112003 (2015).

[17] M. Ablikim et al. (BESIII Collaboration), Phys. Rev. Lett. 115, 222002 (2015).

[18] M. Ablikim et al. (BESIII Collaboration), Phys. Rev. Lett. 113, 212002 (2014).

[19] M. Ablikim et al. (BESIII Collaboration), Phys. Rev. Lett. 115, 182002 (2015).

[20] M. Ablikim et al. (BESIII Collaboration) Phys. Rev. D 91, 112005 (2015).

[21] M. Ablikim et al. (BESIII Collaboration), Phys. Rev. D 86, 071101 (2012).

[22] M. Ablikim et al. (BESIII Collaboration), Phys. Rev. Lett. 114, 092003 (2015).

[23] M. Ablikim et al. (BESIII Collaboration), Phys. Rev. Lett. 118, 092001 (2017).

[24] B. Aubert et al. (BaBar Collaboration), Phys. Rev. Lett. 98, 212001 (2007).

[25] M. Ablikim et al. (BESIII Collaboration), Phys. Rev. D 92, 092006 (2015).

[26] M. Ablikim et al. (BESIII Collaboration), Phys. Rev. Lett. 118, 092002 (2017).

[27] M. Ablikim et al. (BESIII Collaboration), Phys. Rev. Lett. 112, 092001 (2014).

[28] N.A. Törnqvist, Z. Phys. C61, 525 (1994).

[29] M. Karliner, Acta. Phys. Polon. B47, 117 (2016).

[30] R.L. Jaffe, Phys. Rev. D 15, 267 (1977).

[31] D. Horn and J. Mandula, Phys. Rev. D 17, 898 (1978).

[32] N. Isgur, R. Kokoski and J. Paton, Phys. Rev. Lett. 54, 869 (1985).

[33] L. Liu et al. (Hadron Spectrum Collaboration), JHEP 1207, 126 (2012). 
[34] S. Dubynskiy and M.B. Voloshin, Phys. Lett. B666, 344 (2008).

[35] G.-J. Ding, Phys. Rev. D 79, 014001 (20o9).

[36] Q. Wang, C. Hanhart and Q. Zhao, Phys. Rev. Lett. 111, 132003 (2013).

[37] M. Cleven, Q. Wang, F.-K. Guo, C. Hanhart, U.-G. Meissner and Q. Zhao, Phys. Rev. D 90, 074039 (2014).

[38] S.-L. Zhu, Phys. Lett. B625, 22 (2005).

[39] F.E. Close and P.R. Page, Phys. Lett. B628, 215 (2005).

[40] E. Kou and O. Pene, Phys. Lett. B631, 164 (2005).

[41] L. Maiani, F. Piccinini, A.D. Polosa and V. Riquer, Phys. Rev. D 89, 114010 (2014).

[42] H.-X. Chen, L. Maiani, A.D. Polosa and V. Riquer, Eur. Phys. J. C75, 525 (2015).

[43] X. Li and M.B. Voloshin, Mod. Phys. Lett. A29, 2140060 (2014).

[44] M. Ablikim et al. (BESIII Collaboration), in preparation.

[45] N.A. Isgur and M.B. Wise, Phys. Rev. Lett. 66, 1130 (1991).

[46] P. Avery et al. (CLEOII Collaboration), Phys. Lett. B331, 236 (1994).

[47] K. Abe et al. (Belle Collaboration), Phys. Rev. D 69, 112002 (2004).

[48] K. Chilikin et al. (Belle Collaboration), Phys. Rev. D 88, 074026 (2013). 